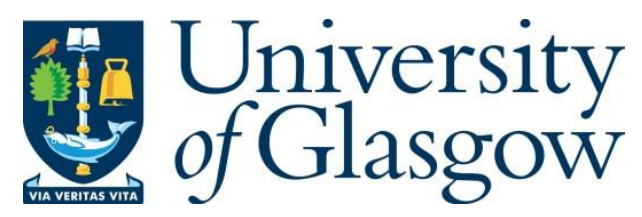

Sodi, R., Eastwood, J., Caslake, M., Packard, C. J., and Denby, L. (2017) Relationship between circulating microRNA-30c with total- and LDL-cholesterol, their circulatory transportation and effect of statins. Clinica Chimica Acta, 466, pp. 13-19.

There may be differences between this version and the published version. You are advised to consult the publisher's version if you wish to cite from it.

http://eprints.gla.ac.uk/134644/

Deposited on: 26 April 2017

Enlighten - Research publications by members of the University of Glasgow http://eprints.gla.ac.uk 


\section{Relationship between Circulating MicroRNA-30c with Total- and LDL- Cholesterol, their Circulatory Transportation and Effect of Statins.}

\section{Ravinder Sodi $^{1,2, *}$, Jarlath Eastwood ${ }^{3}$, Muriel Caslake ${ }^{3}$, Chris J Packard ${ }^{3}$, Laura Denby 4}

1'Department of Biochemistry, Royal Lancaster Infirmary \& Furness General Hospital, University Hospitals of Morecambe Bay NHS Foundation Trust, Lancaster, United Kingdom.

${ }^{2}$ Lancaster Medical School, University of Lancaster, Lancaster, United Kingdom.

${ }^{3}$ Institute of Cardiovascular and Medical Sciences, College of Medical, Veterinary and Life Sciences, University of Glasgow, United Kingdom.

${ }^{4}$ British Heart Foundation Centre for Cardiovascular Science, University of Edinburgh, United Kingdom.

*Address correspondence to this author at:

Department of Biochemistry, Royal Lancaster Infirmary \& Furness

General Hospital, University Hospitals of Morecambe Bay NHS

Foundation Trust, Ashton Road, LA1 4RP, Lancaster, United Kingdom

Email: Ravinder.Sodi@mbht.nhs.uk

Telephone: $+44-7877263296$

Short Title: Circulating miR-30c, lipoprotein cholesterol and effect of statins 
Keywords: circulating microRNA, miR-30c, cholesterol, lipoproteins, statins.

Abbreviations: $\mathrm{Cq}$ - quantification cycle, miR-microRNA, mRNA-messenger RNA, MTP - microsomal triglyceride transfer protein, RT-qPCR - reverse transcription-quantitative real time polymerase chain reaction. 
Background: Small non-coding microRNAs $(\mathrm{miR})$ have important regulatory roles and are used as biomarkers of disease. We investigated the relationship between lipoproteins and circulating miR-30c, evaluated how they are transported in circulation and determined whether statins altered the circulating concentration of miR-30c.

Methods: To determine the relationship between lipoproteins and circulating miR30c, serum samples from 79 subjects recruited from a lipid clinic were evaluated. Ultracentrifugation and nanoparticle tracking analysis was used to evaluate the transportation of miR-30c in the circulation by lipoproteins and extracellular vesicles in three healthy volunteers. Using archived samples from previous studies, the effects of $40 \mathrm{mg}$ rosuvastatin $(n=22)$ and $40 \mathrm{mg}$ pravastatin $(n=24)$ on miR-30c expression was also examined. RNA extraction, reverse transcriptionquantitative real-time polymerase chain reaction was carried out using standard procedures.

Results: When stratified according to total cholesterol concentration, there was increased miR-30c expression in the highest compared to the lowest tertile $(p=0.035)$. There was significant positive correlation between miR-30c and total- $(r=0.367 ; p=$ $0.002)$ and LDL-cholesterol $(r=0.391 ; p=0.001)$. We found that miR-30c was transported in both exosomes and on HDL3. There was a 3.8-fold increased expression of circulating miR-30c after pravastatin treatment for $1-y(p=0.005)$ but no significant change with atorvastatin after 8 -weeks $(p=0.145)$.

Conclusions: This study shows for the first-time in humans that circulating miR-30c is significantly, positively correlated with total- and LDL-cholesterol implicating regulatory functions in lipid homeostasis. We show miR-30c is transported in both exosomes and on HDL3 and pravastatin therapy significantly increased circulating 
miR-30c expression adding to the pleiotropic dimensions of statins. 


\subsection{INTRODUCTION}

Small non-coding microRNAs (miR) are currently a subject of intense research; however, their exact roles in different diseases and in physiological regulation remains incompletely understood. miR are small ( 22 nucleotides), non-coding RNA which regulate gene expression at the post-transcriptional level via inhibition of the translation of messenger RNA (mRNA) or by inducing the degradation of specific mRNA $(1,2)$. In the circulation they are resistant to degradation due in part, to being transported by carrier molecules including lipoproteins such as HDL, proteins such as argonaute-2 and exosomes, which render them highly stable and they can therefore be readily detected in blood samples (3). Thus, they are currently being studied as potential biomarkers of diseases. Circulating levels of specific miRs have been used as biomarkers to diagnose cancers (4); they have been implicated in the pathogenesis of obesity (5), diabetes mellitus (6), coronary artery disease (7) and used in the diagnosis of myocardial infarction (8).

High plasma cholesterol concentration is a known major risk factor for atherosclerosis. Recently, miRs have been linked to cholesterol homeostasis (9). In one study, lentiviral mediated hepatic expression of miR-30c was shown to induce the degradation of microsomal triglyceride transfer protein (MTP) mRNA, which is the apparatus required for the assembly and secretion of apolipoprotein-B containing lipoproteins such as LDL (10). This in turn reduced hyperlipidemia and atherosclerosis in mice without causing hepatosteatosis (10). More recently, the delivery of a miR30c mimic to the liver diminished diet-induced hypercholesterolemia in C57BL/6J mice with long term dose escalation studies showing that miR-30c mimic caused sustained reductions in plasma cholesterol with no obvious side effects (11). In 
addition, miR-30c mimic significantly reduced hypercholesterolemia and atherosclerosis in Apoe ${ }^{-/-}$mice (11). These studies show that miR-30c lowers plasma cholesterol and mitigates atherosclerosis by reducing MTP expression and lipoprotein production without hepatosteatosis. Furthermore, there is emerging evidence that increasing hepatic miR-30c levels may be a viable treatment option for reducing hypercholesterolemia and atherosclerosis.

Whether specific circulating miRs are dysregulated in lipid disorders in humans such as familial hypercholesterolemia $(\mathrm{FH})$ has not been studied before. We therefore hypothesized that circulating miR-30c could reflect tissue level expression and therefore serve as a biomarker of cardiovascular and metabolic diseases. To study this we examined whether circulating miR-30c was correlated with established circulating biomarkers of cardiovascular disease including lipoproteins and cholesterol. In addition, in an effort to understand how miR-30c is transported in circulation as this would affect any observed associations with lipoproteins, we used ultracentrifugation to separate the various lipoprotein fractions to determine in which subfraction they were carried. We used nanoparticle tracking analysis to evaluate the role of extracellular vesicles, specifically exosomes, in trafficking miR-30c. Previous studies have shown that circulating miRs are carried on HDL (12) and to a lesser extent on LDL (13), on proteins such as argonaute 2 (14) and in exosomes (15). However, these studies did not specifically examine miR-30c and there is therefore paucity of data with regard to this. Finally, an emerging concept is whether treatments and interventions alter circulating miR concentrations. Indeed one study by Ortega et al (5) demonstrated that specific circulating miRs changed (some increased whereas others decreased) with bariatric surgery-induced or diet-induced 
weight loss. To date there is limited data regarding the impact of treatment with statins on specific circulating miR concentrations in various population groups. We therefore sought to delineate the effect, if any, of statin therapy on circulating miR30c.

Thus, the aims of this study were: a) determine the relationship between lipoproteins, triglycerides and circulating miR-30c; b) determine how they are transported in circulation; and c) investigate whether statin therapy led to alterations in the circulating concentrations of miR-30c. 


\subsection{MATERIALS AND METHODS}

\subsection{Patients}

To determine the relationship between lipoproteins, triglycerides and circulating miR30c, serum samples from 79 fasted subjects attending the lipid clinic at the Glasgow Royal Infirmary were studied. As this was a proof-of-principle study, we did not analyse outcomes in these patients in any detail and samples were provided to the investigators anonymised. We stratified our cohort into tertiles based on total cholesterol concentrations with those $<5 \mathrm{mmol} / \mathrm{L}$ in the first tertile ('normal' group), those between $5.0-7.5 \mathrm{mmol} / \mathrm{L}$ in the second tertile ('indeterminate group') and those with raised cholesterol $>7.5 \mathrm{mmol} / \mathrm{L}$ in the third tertile ('hypercholesterolemia' group) (16).

To evaluate the transportation of miR-30c in circulation by lipoproteins, we studied serum samples from three healthy volunteers. Samples from two groups of subjects were used to assess the impact of statin therapy on circulating miR-30c concentration. For the study involving rosuvastatin all the available remnant samples ( $n=22$ from the original 29) were used (17). In this study, subjects with raised cholesterol were used in a cross-over trial to evaluate rosuvastatin $40 \mathrm{mg} / \mathrm{d}$. After a 5 week dietary run-in subjects were randomized to treatment or placebo for 8 weeks, followed by a 4 week washout period and then crossed over to the opposite arm of the study for a further 8 weeks. The miR concentrations were determined in stored (frozen at $-80^{\circ} \mathrm{C}$ ) samples from baseline and the end of each treatment period. The second group was 24 men from the active arm (i.e. on pravastatin 40 $\mathrm{mg} / \mathrm{d}$ ) of the West of Scotland Coronary Prevention Study (WOSCOPS) (18), from whom stored samples were available for the baseline and one-year time-points. Again 
the samples had been stored at $-80^{\circ} \mathrm{C}$. For this study, samples were randomly selected by the laboratory technician from the available samples stored in our institution using the random number generator function of Excel.

The local Research Ethics Committee approved this study and all volunteers in the original studies gave informed written consent. All procedures were conducted in accordance with the guidelines of The Declaration of Helsinki.

\subsection{Lipid measurements}

All analysis was carried out on the ILAB 600 Clinical Chemistry Analyser, using Roche reagents (Burgess Hill, UK). Total-cholesterol, HDL-cholesterol and triglyceride were measured by enzymatic-colorimetric methods whereas LDL was calculated using the Friedewald equation, where LDL-cholesterol $=$ Total cholesterol HDL cholesterol - (Triglycerides/2.2) and is only valid when the triglyceride concentration is less than $4.6 \mathrm{mmol} / \mathrm{L}$ or Triglycerides $/ 5.0$ if cholesterol and triglycerides are reported in $\mathrm{mg} / \mathrm{dL}$ (to convert Triglyceride $\mathrm{mg} / \mathrm{dL}$ to $\mathrm{mmol} / \mathrm{L}$, multiply by 88.5$)$. VLDL-cholesterol ( $\mathrm{mmol} / \mathrm{L})$ was estimated by dividing the triglycerides by 2.2.

\subsection{Separation of lipoprotein subfractions by centrifugation}

To evaluate how miR-30c is transported in the circulation, serum samples were collected into clot activator tubes with gel separator from three healthy volunteers. The samples were centrifuged at $10,000 \mathrm{~g}$ for 10 minutes at a temperature of $4^{\circ} \mathrm{C}$. The resultant supernatant was then separated into lipid subfractions by sequential centrifugation using density gradient solutions on the Beckman TLA 100.2 
ultracentrifuge (Beckman Coulter, UK). Briefly, VLDL was first separated by overlaying $500 \mu \mathrm{L}$ of sample with $500 \mu \mathrm{L}$ of $1.006 \mathrm{~g} / \mathrm{mL}$ density solution and centrifuged at $100,000 \mathrm{~g}$ for 2.5 hours at $25^{\circ} \mathrm{C}$. To isolate IDL, $500 \mu \mathrm{L}$ of the remaining infranatant from the previous step was mixed with $40 \mu \mathrm{L}$ of $1.182 \mathrm{~g} / \mathrm{mL}$ density solution and overlaid with $460 \mu \mathrm{L}$ of $1.019 \mathrm{~g} / \mathrm{mL}$ density solution and centrifuged at $100,000 \mathrm{~g}$ for 2.5 hours at $25^{\circ} \mathrm{C}$. To isolate LDL, $500 \mu \mathrm{L}$ of the remaining infranatant was mixed with $184 \mu \mathrm{L}$ of $1.182 \mathrm{~g} / \mathrm{mL}$ density solution and then overlaid with $316 \mu \mathrm{L}$ of $1.063 \mathrm{~g} / \mathrm{mL}$ of density solution. This was centrifuged as above. To isolate HDL2, $500 \mu \mathrm{L}$ infranatant was mixed with $88 \mu \mathrm{L}$ of $1.478 \mathrm{~g} / \mathrm{mL}$ of density solution and then overlaid with $412 \mu \mathrm{L}$ of $1.125 \mathrm{~g} / \mathrm{mL}$ density solution, centrifuged at $53,000 \mathrm{~g}$ for 18 hours at $23^{\circ} \mathrm{C}$. Finally to isolate HDL3, $500 \mu \mathrm{L}$ infranatant was mixed with $160 \mu \mathrm{L}$ of $1.478 \mathrm{~g} / \mathrm{mL}$ density solution and then overlaid with $340 \mu \mathrm{L}$ of $1.21 \mathrm{~g} / \mathrm{mL}$ of density solution. This was then centrifuged at $100,000 \mathrm{~g}$ for 5 hours at $23^{\circ} \mathrm{C}$. The remaining infranatant was deemed to be lipid depleted (LPDS), which was confirmed by the demonstration of negligible concentrations of lipids and apolipoproteins in them (Supplemental Table 1). At each of the above steps, $500 \mathrm{uL}$ supernatant was stored at $-80^{\circ} \mathrm{C}$ for the subsequent extraction of RNA.

\subsection{Nanoparticle tracking analysis to determine presence of extracellular} vesicles

Given that a large percentage of the miRs studied were detected in the lipid-depleted subfraction (LPDS), we inferred that they contained extracellular vesicles. To confirm this we used nanoparticle tracking analysis (NTA) performed with a NanoSight LM10 instrument (NanoSight Ltd, Amesbury, UK). NTA captures real-time videos, showing particles moving by Brownian motion (19). A $635 \mathrm{~nm}$ laser is used to illuminate nanoparticles in liquid suspension. The light scattered from the particles can be 
visualized using a $\times 20$ objective lens of a light microscope that is attached to a camera running at 30 frames per second. The speed of moving particles is determined on a particle-by-particle basis, and the size is calculated using the Stokes-Einstein equation (19). A video of 60 seconds duration was recorded and data analysis was carried out using the NTA analytical software version 2.3 (NanoSight Ltd, UK). We selected particles of size between 30 and $120 \mathrm{~nm}$, which are known to be specific for exosomes (15).

\subsection{RNA extraction}

Total RNA including miRs was isolated using the commercial miRNeasy ${ }^{\mathrm{TM}}$ kit (QIAGEN, Germany) using $200 \mu \mathrm{L}$ serum from each patient. All samples were spiked with $50 \mathrm{pmol} / \mathrm{L}$ of synthesized Caenorhabditis elegans miR - cel-miR-39-3p (Applied Biosystems/Thermo-Fisher Scientific, UK) - that lacked sequence homology to human miRs. This was used to serve as an internal control to monitor extraction efficiency and used for data normalisation. According to the manufacturer (QIAGEN, Germany), DNAse treatment is not recommended for serum or plasma samples as the combined QIAzol ${ }^{\mathrm{TM}}$ and RNeasy ${ }^{\mathrm{TM}}$ technologies efficiently removes the trace amount of DNA. The extracted RNA was eluted into RNAse free water provided in the kit and all samples were adjusted to a fixed total RNA concentration of $2 \mathrm{ng} / \mu \mathrm{L}$ prior to further use. The final RNA yield and purity was determined by the A260/A280 ratio measured by a NanoDrop ND-1000 spectrophotometer (NanoDrop Technologies/Thermo-Fisher Scientific, UK) with a ratio 1.70 to 2.00 indicative of good RNA purity. The isolated RNA was stored at $-80^{\circ} \mathrm{C}$ for further use. 


\subsection{Reverse transcription (RT)}

The extracted RNA was reverse transcribed using a commercially available kit (Applied Biosystems/Thermo-Fisher Scientific, UK) using miR-specific stem-loop primers as per manufacturer instructions. $5 \mu \mathrm{L}$ of the sample was added to a 96-well plate together with $10 \mu \mathrm{L}$ reaction mixture (MasterMix ${ }^{\mathrm{TM}}$ ) containing $1.50 \mu \mathrm{L} 10 \mathrm{X}$

reverse transcription buffer, $0.15 \mu \mathrm{L}$ dNTP $(100 \mathrm{mM}), 1.00 \mu \mathrm{L}$ Multiscribe ${ }^{\mathrm{TM}}$ reverse transcriptase $(50 \mathrm{U} / \mu \mathrm{L}), 0.19 \mu \mathrm{L}$ RNAase inhibitor $(20 \mathrm{U} / \mu \mathrm{L}), 4.16 \mu \mathrm{L}$ nuclease-free water and $3 \mu \mathrm{L} 5 \mathrm{X}$ specific RT primers. The RT was carried out in a PTC-225 Peltier Thermal Cycler (MJ Research Inc., Waltham, Massachusetts) at $16^{\circ} \mathrm{C}$ for $30 \mathrm{~min}$, then $42^{\circ} \mathrm{C}$ for 30 mins, followed by $85^{\circ} \mathrm{C}$ for 5 mins and then kept at $4^{\circ} \mathrm{C}$. A no template control (NTC) was included in each experiment to account for contamination by genomic DNA. The negative control produced no detectable signal in any of the experiments. In all cases, the RT product was quantified immediately by qPCR and the remainder stored at $-80^{\circ} \mathrm{C}$.

\subsection{Gene expression by quantitative real time PCR (qPCR)}

The miRs were quantified using TaqMan ${ }^{\mathrm{TM}}$ MicroRNA assays (Applied Biosystems/Thermo-Fisher Scientific, UK) in a 384 well plate in duplicate. We used 0.7 $\mu \mathrm{L}$ of the RT product, 5.0 $\mu \mathrm{L}$ TaqMan $^{\mathrm{TM}}$ Universal PCR MasterMix II - no UNG, (Applied Biosystems/Thermo-Fisher Scientific, UK), 3.8 $\mu \mathrm{L}$ RNAse free water and $5 \mu \mathrm{L}$ 20X TaqMan ${ }^{\text {TM }}$ MicroRNA assay. The qPCR was performed using the $7900 \mathrm{HT}$ Fast Real-Time PCR System (Applied Biosystems, UK) and manually set-up as follows: $95^{\circ} \mathrm{C}$ for 10 mins followed by 40 cycles of $95^{\circ} \mathrm{C}$ for $15 \mathrm{~s}$ and $60^{\circ} \mathrm{C}$ for $60 \mathrm{~s}$. The 
quantification cycle $(\mathrm{Cq})$ was determined with instrument default threshold settings (10 SDs above the mean fluorescence of the baseline cycle).

All experimental work was carried out in accordance with the Minimum Information about Quantitative Real-Time Polymerase Chain Reaction Experiments (MIQE) (20). All primer sequences used in this study are given in Supplemental Table 2. The precision of the experiments in this study were determined using a nested analysis of variation (ANOVA) model (21) and the data is shown in Supplemental Table 3.

\subsection{Statistical analysis and data normalization}

The use of a fixed concentration of total RNA in all experiments allowed for direct comparisons of the miR expression data across the groups. Quantification cycle $(\mathrm{Cq})$ and miR copy number are reciprocal and related by a log2 factor implying a change in $\mathrm{Cq}$ of 1 represents a 2 -fold change in miR copy number. In addition, the lower the $\mathrm{Cq}$, the higher the miR expression. Data were normalised by determining $\Delta \mathrm{Cq}$, which is the difference between the expression of the control miR-39 and miR-30c. Relative expression was determined in relation to changes over control $(\Delta \Delta \mathrm{Cq})$. The data were normally distributed according to the Kolmogorov-Smirnov test and as such associations were determined using Pearson correlation. For the comparison of more than two normally distributed variables, one-way ANOVA followed by Tukey pairwise comparison was undertaken. For the comparison of paired data, paired ttest was used. Statistical analyses were undertaken using Minitab version 17.1.0. 


\subsection{RESULTS}

\subsection{Association of circulating miR-30c with serum lipids.}

Table 1 shows the laboratory characteristics of the study sample stratified into tertiles according to total cholesterol concentration as described in the methods. Of the 79 subjects studied there were insufficient sample or spoilt analyses in 9 cases. Of the remaining 70 subjects, when stratified according to total cholesterol concentration, there was significantly increased miR-30c expression in the group with highest compared to the lowest tertile (Figure $1 \mathrm{~A}, \mathrm{~F}=3.27 ; \mathrm{p}=0.044$; the $\mathrm{p}$-values by Tukey multiple comparison are shown on the graph). When stratified according to LDLcholesterol there was also significantly increased miR-30c expression in the group with the highest compared to the lowest tertile (Figure $1 B, F=3.62 ; p=0.032$; the $p$ values by Tukey multiple comparison are shown on the graph). In addition, Figure 2 shows there was significant positive correlation between miR-30c and total cholesterol $(r=0.367 ; p=0.002)$ and LDL-cholesterol $(r=0.391 ; p=0.001)$.

\subsection{Circulating miR-30c transportation}

To account for the mode of transportation in circulation, we studied the roles of lipoproteins and extracellular vesicles. Figure 3A shows the relative expression of miR-30c in each of the lipoprotein and lipid depleted (LPDS) sub-fractions. LPDS and HDL3 showed significantly higher relative miR-30c expression $(\Delta \mathrm{Cq}=14.0 \pm 0.7$ and 13.8 \pm 0.2 , respectively $)$ compared to HDL2 $(\Delta \mathrm{Cq}=17.1 \pm 0.3), \operatorname{LDL}(\Delta \mathrm{Cq}=19.6$ $\pm 0.6)$, IDL $\left(\Delta \mathrm{C}_{q}=18.3 \pm 0.3\right)$ and VLDL $\left(\Delta \mathrm{C}_{q}=18.8 \pm 0.3\right)(F=32.54 ; p<0.0001)$ and were not significantly different from each other. We confirmed the presence of exosomes in LPDS as shown in Figure 3B. The mean (SD) particle size was 47 (32) $\mathrm{nm}$ and the mode was $32 \mathrm{~nm}$, which was the expected size range for exosomes (30- 
$120 \mathrm{~nm}$ as shown by vertical demarcations). The mean concentration in a

representative experiment was $23.3 \times 10^{8}$ exosomes $/ \mathrm{mL}$. These data reveal that miR$30 \mathrm{c}$ is present in circulation mainly in exosomes and bound to HDL3 with negligible amounts trafficked on other lipoproteins.

\subsection{Circulating miR-30c and effect of statin therapy}

Treatment with pravastatin significantly increased mean circulating miR-30c expression as shown in Figure $4 \mathrm{~A}(\Delta$ Cqbefore $=10.8 \pm 0.3 ; \Delta$ Cqafter $=9.8 \pm 0.2$; $p=0.005$ ), representing an overall 3.8 -fold increase in expression across the subjects studied. Of the 24 subjects, 19 showed an increased expression in circulating miR30c as denoted by a decrease in $\Delta \mathrm{C}_{\mathrm{q}}$ (Figure 4B). Rosuvastatin treatment also increased circulating miR-30c expression (15 of 22 subjects) as shown in Figure 4A and $4 \mathrm{C}(\Delta \mathrm{Cqbefore}=17.5 \pm 0.3 ; \Delta \mathrm{Cqafter}=16.9 \pm 0.3)$ but the change in expression was not found to be statistically significant $(p=0.145)$. 


\subsection{DISCUSSION}

This study shows for the first time a positive correlation between miR-30c and totaland LDL-cholesterol in humans. We found that miR-30c is transported in both exosomes and by HDL3. In addition, we showed that pravastatin therapy significantly increased miR-30c.

\subsection{Circulating miR-30c and regulation of cholesterol homeostasis}

We observed a significant, positive correlation between miR-30c and total- and LDLcholesterol. In addition, we found that those with a total cholesterol $>7.5 \mathrm{mmol} / \mathrm{L}$ had a significantly higher miR-30c than those with $<5.0 \mathrm{mmol} / \mathrm{L}$. To the best of our knowledge, this is the first such report in humans. A previous study in mice (10) showed that miR-30c induced the degradation of MTP mRNA leading to decreased secretion of apolipoprotein B. In addition, independently of MTP, miR-30c reduced hepatic lipid synthesis eventually attenuating hyperlipidemia and consequently mitigating atherosclerosis in animal models (10). A tentative interpretation of our finding of increased expression of miR-30c as cholesterol rises is that it is a compensatory mechanism activated to decrease the secretion of both lipids and lipoproteins when circulating levels are high. It has been suggested that raising miR30c levels might be useful in the treatment of hyperlipidemia and associated atherosclerosis $(10,11)$. The idea that miR-30c may be used as a biomarker for lipid disorders such as $\mathrm{FH}$ is also an interesting prospect. Given that we showed a clear demarcation between those with normal total cholesterol concentrations compared to concentrations expected in patients with lipid disorders such as $\mathrm{FH}$, further work in large, unselected populations is required to confirm this observation. 


\subsection{Circulating miR-30c transportation}

It is known that miR circulate bound to HDL (12) and to a lesser extent to LDL particles (13). However, to the best of our knowledge there is no specific information on the lipoprotein-mediated transport of miR-30c. The idea that exosomes offer an important mode of intracellular communication is an area of growing interest (22). Indeed one landmark study has shown exosomes deliver specific miR via the circulation and protect the myocardium from ischemia-reperfusion injury (23) whereas another has shown that miRs in exosomes can be therapeutically manipulated in circulation to reduce in-stent restenosis (24). Our finding that miR$30 \mathrm{c}$ is shuttled both in exosomes and by HDL3 opens up the possibility that it may be used therapeutically, either by strategies that increase HDL concentrations or by manipulating the exosomes that shuttle them. This study also reveals for the first time yet another role for the small dense HDL molecule - HDL3 in the transportation of miR-30c. It is noteworthy that low HDL3 has been shown to be independently associated with a greater risk of myocardial infarction (25). The finding of this study that miR-30c is shuttled by HDL3 but yet attenuates total and LDL-cholesterol remains to be reconciled. Whether other miRs are carried by HDL3 and the implications of that remains to be established. Whether these miRs are responsible for the functions of the HDL particles they are carried on remains to be explored. We did not account for protein-bound concentrations of circulating miR-30c (14) but this is a constant and would not affect the results of this study.

\subsection{Circulating miR-30c and effect of statin therapy}

It is well known that statins exert benefits beyond lipid-lowering effects including blood-pressure lowering and cardiac remodelling (26). Furthermore, it is becoming 
clear that lipophilic statins such as atorvastatin might be more potent than hydrophilic statins such as pravastatin and rosuvastatin (27). In this study, we showed that pravastatin but not rosuvastatin significantly increased circulating miR-30c concentration. This might suggest a differential therapeutic effect as both drugs are hydrophilic. However, it should be noted that the period of treatment varies considerably - 1 year with pravastatin compared to 8 weeks with rosuvastatin, and it may take time for the statins to exert an effect on miR-30c expression. The pleiotropic effects of statins are a theme of current research (28), and our finding that statins augment circulating miR-30c adds to the list of their potential beneficial effects. One study (29) examined Chinese patients with unstable angina and sought to determine how statin therapy affected their circulating miRs. They found that statins induced a significant up-regulation of a number of circulating miR including miR-30c, which was increased by 2.1 -fold, an observation that is in concordance with the findings of this study in a primarily Caucasian population. The mechanism(s) of how statins alter circulating miR and the interplay between liver production and circulating miR remains to be established. We speculate that this paradoxical effect of pravastatin that decreases LDL-cholesterol but yet increases the expression of miR30c might be an attempt to independently augment the decreased synthesis of lipoproteins in the face of hyperlipidemia but further experimental work is required to confirm this hypothesis.

\subsection{Strengths and Limitations of this Study}

The strengths of the study are the use of a non-human internal control. The normalisation of data derived from studies involving miR is a subject of current debate (30). Commonly, studies investigating miR use a housekeeper miR known to 
be present in the sample, but there is now evidence that these can change due to disease (31). The use of an internal standard not expressed in humans such as celmiR-39-3p alleviates this problem (32). In addition, we used a fixed total RNA concentration in all experiments allowing for comparisons across groups. The main limitation of this study was the sample size and use of archived samples. Archived samples provide an invaluable opportunity to test hypotheses. Further work with a larger sample size and diverse population groups is required to explore the effects of statins but the results reported here are revelatory.

\subsection{Conclusions}

This study has shown for the first time that in humans circulating miR- $30 \mathrm{c}$ is significantly correlated with total- and LDL-cholesterol implicating possible regulatory functions in lipid homeostasis. We show that miR-30c is carried in both exosomes and on HDL3 and pravastatin therapy significantly increased circulating miR-30c expression adding to the pleiotropic dimensions of statins and alluding to the possibility of therapeutic manipulation. 
Contributions - All authors have:

i) made a substantial contribution to the concept and design, acquisition of data or analysis and interpretation of data;

ii) drafted the article or revised it critically for important intellectual content;

iii) approved the version to be published.

Research Funding: LD is in receipt of a Kidney Research UK fellowship PD6/2012 and has a PhD studentship co-sponsored by Regulus Therapeutics. Part of this work was funded by Medical Research Scotland Scholarship to JE.

\section{Authors' Disclosures or Potential Conflicts of Interest -}

Employment or Leadership: None declared.

Consultant or Advisory Role: None declared.

Stock Ownership: None declared.

Honoraria: None declared.

Expert Testimony: None declared.

Role of Sponsor: The funding organizations played no role in the design of the study, review and interpretation of data, or preparation or approval of manuscript. Acknowledgements: We acknowledge the technical assistance of Josephine Clooney and James Philip Stewart, Institute of Cardiovascular and Medical Sciences, University of Glasgow. We thank the West of Scotland Coronary Prevention Study (WOSCOPS) committee and Astra Zeneca for providing samples from their respective study cohorts. 


\section{References}

1. Bartel DP. MicroRNAs: genomics, biogenesis, mechanism, and function. Cell. $2004 ; 116(2): 281-97$.

2. Ambros V. The functions of animal microRNAs. Nature. 2004;431(7006):350-5.

3. Mitchell PS, Parkin RK, Kroh EM, Fritz BR, Wyman SK, PogosovaAgadjanyan EL, et al. Circulating microRNAs as stable blood-based markers for cancer detection. Proc Natl Acad Sci U S A. 2008;105(30):10513-8.

4. Ueda T, Volinia S, Okumura H, Shimizu M, Taccioli C, Rossi S, et al. Relation between microRNA expression and progression and prognosis of gastric cancer: a microRNA expression analysis. Lancet Oncol. 2010;11(2):136-46.

5. Ortega FJ, Mercader JM, Catalan V, Moreno-Navarrete JM, Pueyo N, Sabater $\mathrm{M}$, et al. Targeting the circulating microRNA signature of obesity. Clin Chem. 2013;59(5):781-92.

6. Zampetaki A, Kiechl S, Drozdov I, Willeit P, Mayr U, Prokopi M, et al. Plasma microRNA profiling reveals loss of endothelial miR-126 and other microRNAs in type 2 diabetes. Circ Res. 2010;107(6):810-7.

7. Fichtlscherer S, De Rosa S, Fox H, Schwietz T, Fischer A, Liebetrau C, et al. Circulating microRNAs in patients with coronary artery disease. Circ Res. 2010;107(5):677- 84 .

8. Devaux Y, Vausort M, Goretti E, Nazarov PV, Azuaje F, Gilson G, et al. Use of circulating microRNAs to diagnose acute myocardial infarction. Clin Chem. 2012;58(3):559- 67.

9. Goedeke L, Aranda JF, Fernandez-Hernando C. microRNA regulation of lipoprotein metabolism. Curr Opin Lipidol. 2014;25(4):282-8.

10. Soh J, lqbal J, Queiroz J, Fernandez-Hernando C, Hussain MM. MicroRNA$30 \mathrm{c}$ reduces hyperlipidemia and atherosclerosis in mice by decreasing lipid 
synthesis and lipoprotein secretion. Nat Med. 2013;19(7):892-900.

11. Irani S, Pan X, Peck BC, lqbal J, Sethupathy P, Hussain MM. MicroRNA30c mimic mitigates hypercholesterolemia and atherosclerosis in mice. J Biol Chem. 2016.

12. Vickers KC, Palmisano BT, Shoucri BM, Shamburek RD, Remaley AT. MicroRNAs are transported in plasma and delivered to recipient cells by highdensity lipoproteins. Nat Cell Biol.2011;13(4):423-33.

13. Wagner J, Riwanto M, Besler C, Knau A, Fichtlscherer S, Roxe T, et al. Characterization of levels and cellular transfer of circulating lipoprotein-bound microRNAs. Arterioscler Thromb Vasc Biol. 2013;33(6):1392-400.

14. Arroyo JD, Chevillet JR, Kroh EM, Ruf IK, Pritchard CC, Gibson DF, et al. Argonaute2 complexes carry a population of circulating microRNAs independent of vesicles in human plasma. Proc Natl Acad Sci U S A. 2011;108(12):5003-8.

15. Chevillet JR, Kang Q, Ruf IK, Briggs HA, Vojtech LN, Hughes SM, et al. Quantitative and stoichiometric analysis of the microRNA content of exosomes. Proc Natl Acad Sci U S A. 2014;111(41):14888-93.

16. Risk of fatal coronary heart disease in familial hypercholesterolaemia. Scientific Steering Committee on behalf of the Simon Broome Register Group. BMJ. 1991;303(6807):893-6.

17. Caslake MJ, Stewart G, Day SP, Daly E, McTaggart F, Chapman MJ, et al. Phenotype-dependent and -independent actions of rosuvastatin on atherogenic lipoprotein subfractions in hyperlipidaemia. Atherosclerosis. 2003;171(2):245-53.

18. Shepherd J, Cobbe SM, Ford I, Isles CG, Lorimer AR, MacFarlane PW, et al. Prevention of coronary heart disease with pravastatin in men with hypercholesterolemia. West of Scotland Coronary Prevention Study Group. N Engl J Med. 1995;333(20):1301-7. 
19. Dragovic RA, Gardiner C, Brooks AS, Tannetta DS, Ferguson DJ, Hole P, et al. Sizing and phenotyping of cellular vesicles using Nanoparticle Tracking Analysis. Nanomedicine. 2011;7(6):780-8.

20. Bustin SA, Benes V, Garson JA, Hellemans J, Huggett J, Kubista M, et al. The MIQE guidelines: minimum information for publication of quantitative real-time PCR experiments. Clin Chem. 2009;55(4):611-22.

21. Tichopad A, Kitchen R, Riedmaier I, Becker C, Stahlberg A, Kubista M. Design and optimization of reverse-transcription quantitative PCR experiments. Clin Chem. 2009;55(10):1816-23.

22. Valadi H, Ekstrom K, Bossios A, Sjostrand M, Lee JJ, Lotvall JO. Exosomemediated transfer of mRNAs and microRNAs is a novel mechanism of genetic exchange between cells. Nat Cell Biol. 2007;9(6):654-9.

23. Vicencio JM, Yellon DM, Sivaraman V, Das D, Boi-Doku C, Arjun S, et al. Plasma exosomes protect the myocardium from ischemia-reperfusion injury. $\mathrm{J}$ Am Coll Cardiol. 2015;65(15):1525-36.

24. McDonald RA, Halliday CA, Miller AM, Diver LA, Dakin RS, Montgomery J, et al. Reducing in-stent restenosis: therapeutic manipulation of miRNA in vascular remodeling and inflammation. J Am Coll Cardiol. 2015;65(21):2314-27.

25. Martin SS, Khokhar AA, May HT, Kulkarni KR, Blaha MJ, Joshi PH, et al. HDL cholesterol subclasses, myocardial infarction, and mortality in secondary prevention: the Lipoprotein Investigators Collaborative. Eur Heart J. 2015;36(1):2230.

26. Strazzullo P, Kerry SM, Barbato A, Versiero M, D'Elia L, Cappuccio FP. Do statins reduce blood pressure?: a meta-analysis of randomized, controlled trials. Hypertension. 2007;49(4):792-8.

27. Andersson C, Vasan RS. Lipophilic Statins and Aldosterone Secretion: A 
Bridge Too Far? Circulation. 2015;132(19):1783-5.

28. Bhandari S, Gupta P, Quinn P, Sandhu J, Hakimi A, Jones D, et al. Pleiotropic effects of statins in hypercholesterolaemia: a prospective observational study using a lipoproteomic based approach. Lancet. 2015;385 Suppl 1:S21.

29. Li J, Chen H, Ren J, Song J, Zhang F, Zhang J, et al. Effects of statin on circulating microRNAome and predicted function regulatory network in patients with unstable angina. BMC Med Genomics. 2015;8:12.

30. Schwarzenbach $\mathrm{H}$, da Silva AM, Calin G, Pantel K. Data normalization strategies for microRNA quantification. Clin Chem. 2015;61(11):1333-42.

31. Creemers EE, Tijsen AJ, Pinto YM. Circulating microRNAs: novel biomarkers and extracellular communicators in cardiovascular disease? Circ Res. $2012 ; 110(3): 483-95$

32. McDonald JS, Milosevic D, Reddi HV, Grebe SK, Algeciras-Schimnich A. Analysis of circulating microRNA: preanalytical and analytical challenges. Clin Chem. $2011 ; 57(6): 833-40$. 
Table 1. Laboratory characteristics of participants stratified according to total cholesterol concentration. Mean \pm standard deviation shown. Of 79 subjects studied, there was incomplete data in 9 cases giving a total of 70 across the tertiles.

\begin{tabular}{|c|c|c|c|c|c|}
\hline & $\begin{array}{l}\text { All subjects } \\
(n=70)\end{array}$ & $\begin{array}{l}\text { Tertile } 1 \\
(\mathrm{n}=16)\end{array}$ & $\begin{array}{l}\text { Tertile } 2 \\
(n=37)\end{array}$ & $\begin{array}{l}\text { Tertile } 3 \\
(\mathrm{n}=17)\end{array}$ & $\mathrm{p} \mathrm{value}^{\dagger}$ \\
\hline Total-cholesterol $(\mathrm{mmol} / \mathrm{L})^{\mathrm{a}}$ & $6.36 \pm 1.5$ & $4.33 \pm 0.4^{*} \ddagger$ & $6.26 \pm 0.7^{*} \Phi$ & $8.33 \pm 0.6 \pm T$ & $<0.0001$ \\
\hline LDL-cholesterol $(\mathrm{mmol} / \mathrm{L})^{a}$ & $3.96 \pm 1.2$ & $2.43 \pm 0.3^{*} \ddagger$ & $3.87 \pm 0.8^{*} \mathbb{T}$ & $5.51 \pm 0.5 \pm \pi$ & $<0.0001$ \\
\hline HDL-cholesterol $(\mathrm{mmol} / \mathrm{L})^{\mathrm{a}}$ & $1.43 \pm 0.4$ & $1.00 \pm 0.2^{*} \ddagger$ & $1.51 \pm 0.4^{*} \mathbb{T}$ & $1.62 \pm 0.4 \mp \mathrm{T}$ & $<0.0001$ \\
\hline VLDL-cholesterol $(\mathrm{mmol} / \mathrm{L})^{\mathrm{a}}$ & $0.97 \pm 0.3$ & $0.96 \pm 0.2 \ddagger$ & $0.91 \pm 0.2 \rrbracket$ & $1.20 \pm 0.3 \mp T$ & 0.001 \\
\hline Triglycerides $(\mathrm{mmol} / \mathrm{L})^{b}$ & $1.31 \pm 0.4$ & $1.31 \pm 0.4$ & $1.23 \pm 0.3$ & $1.42 \pm 0.4$ & 0.201 \\
\hline
\end{tabular}

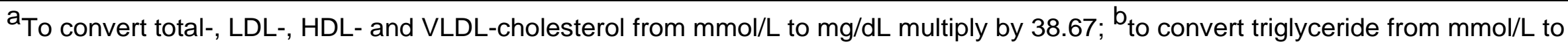
$\mathrm{mg} / \mathrm{dL}$ multiply by $88.57{ }^{\dagger}$ ANOVA to test differences in the means between the tertiles followed by Tukey multiple comparison with significant statistical difference shown by * Tertile 1 vs 2 ; † tertile 1 vs 3 ; ๆ Tertile 2 vs 3. 
Figure 1. miR-30c expression stratified as tertiles according to total-cholesterol (A) and LDL-cholesterol (B) concentrations. Total cholesterol: tertile $1<5.0 \mathrm{mmol} / \mathrm{L}$ ('normal group'; $\mathrm{n}=16$ ); tertile $2=5.0-7.5$ $\mathrm{mmol} / \mathrm{L}$ ('indeterminate group'; $\mathrm{n}=37$ ); tertile $3>7.5 \mathrm{mmol} / \mathrm{L}$ ('hypercholesterolemia group'; $\mathrm{n}=17$ ). $\mathrm{RQ}$ - relative quantification following log2 transformation of data. Mean (SE) shown. The p-values by Tukey multiple comparisons are shown on the graph.

Figure 2. Correlation of miR-30c with lipoproteins and triglyceride. Chol - cholesterol, LDL - low density lipoprotein, HDL - high density lipoprotein, VLDL - very low density lipoprotein, Trig - triglycerides all given on the $\mathrm{y}$ axis. The inverse delta $\mathrm{Cq}$ is shown on the $\mathrm{x}$ axis. The inverse is shown for clarity because the higher the $\mathrm{Cq}$ the lower the miR expression. All measured in $\mathrm{mmol} / \mathrm{L}$.

Figure 3. A) Expression of miR-30c in various lipoprotein sub-fractions relative to respective unseparated serum. $R Q$ - relative quantification following log2 transformation of data. LPDS - lipiddepleted sub-fraction, HDL - high density lipoprotein-cholesterol, LDL - low density lipoproteincholesterol, IDL - intermediate density lipoprotein-cholesterol, VLDL - very low density lipoproteincholesterol. * LPDS and HDL3 showed significantly higher relative expression compared to the other lipoproteins. Mean (SE) shown. B) The presence of exosomes (30-120 nm) in the LPDS. Particle size is shown against relative intensity (arbitrary units, AU). Inset - representative video frame of exosomes. Figure 4. Effect of $40 \mathrm{mg}$ Pravastatin $(n=24$; Figures $A \& B$ ) and 40 mg Rosuvastatin $(n=22 ;$ Figures A \& C) on circulating miR-30c expression. Figure A shows mean (SE) fold-change whereas $B$ \& $C$ show individual $\Delta \mathrm{Cq}$ before and after treatment 


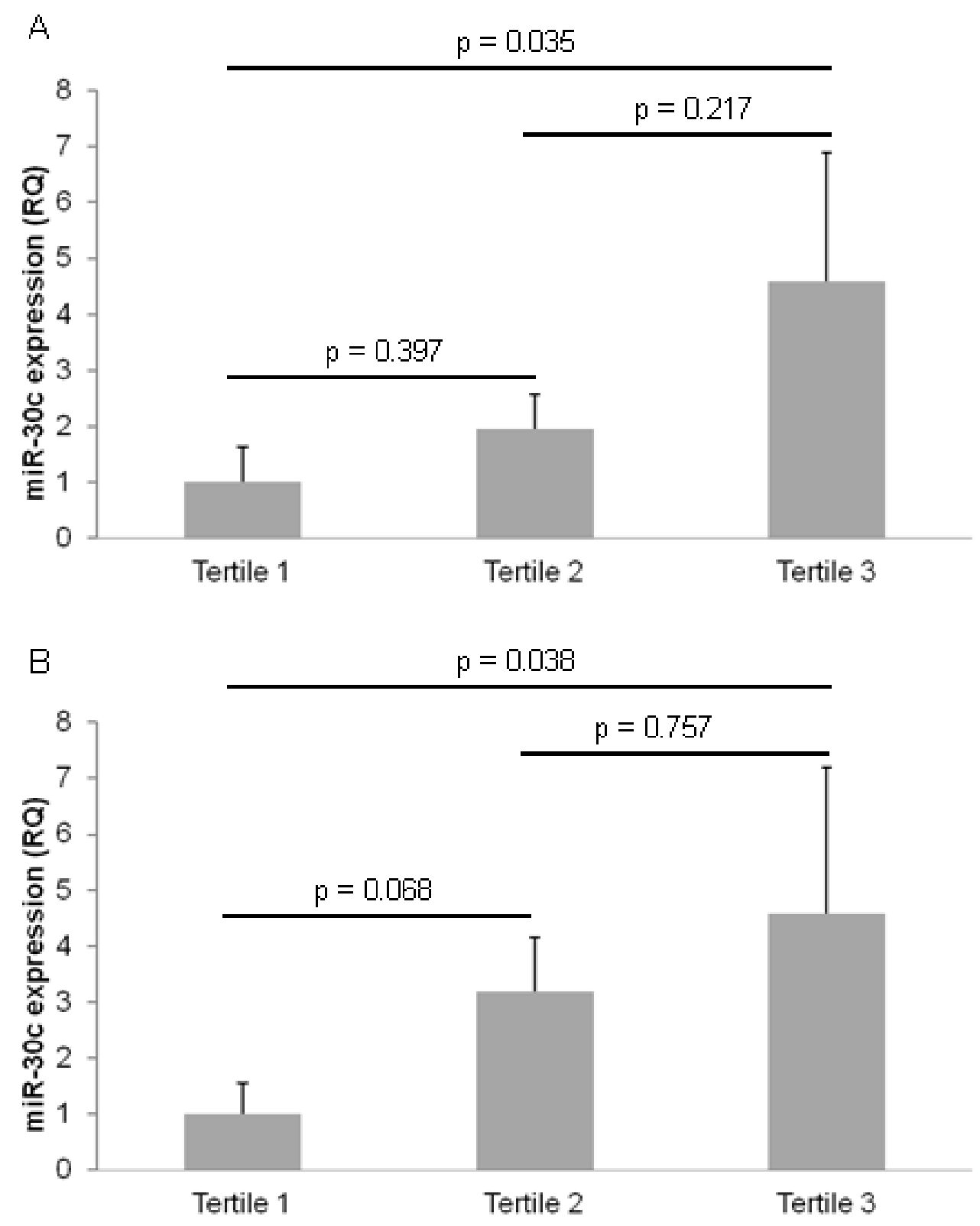

Figure 1 

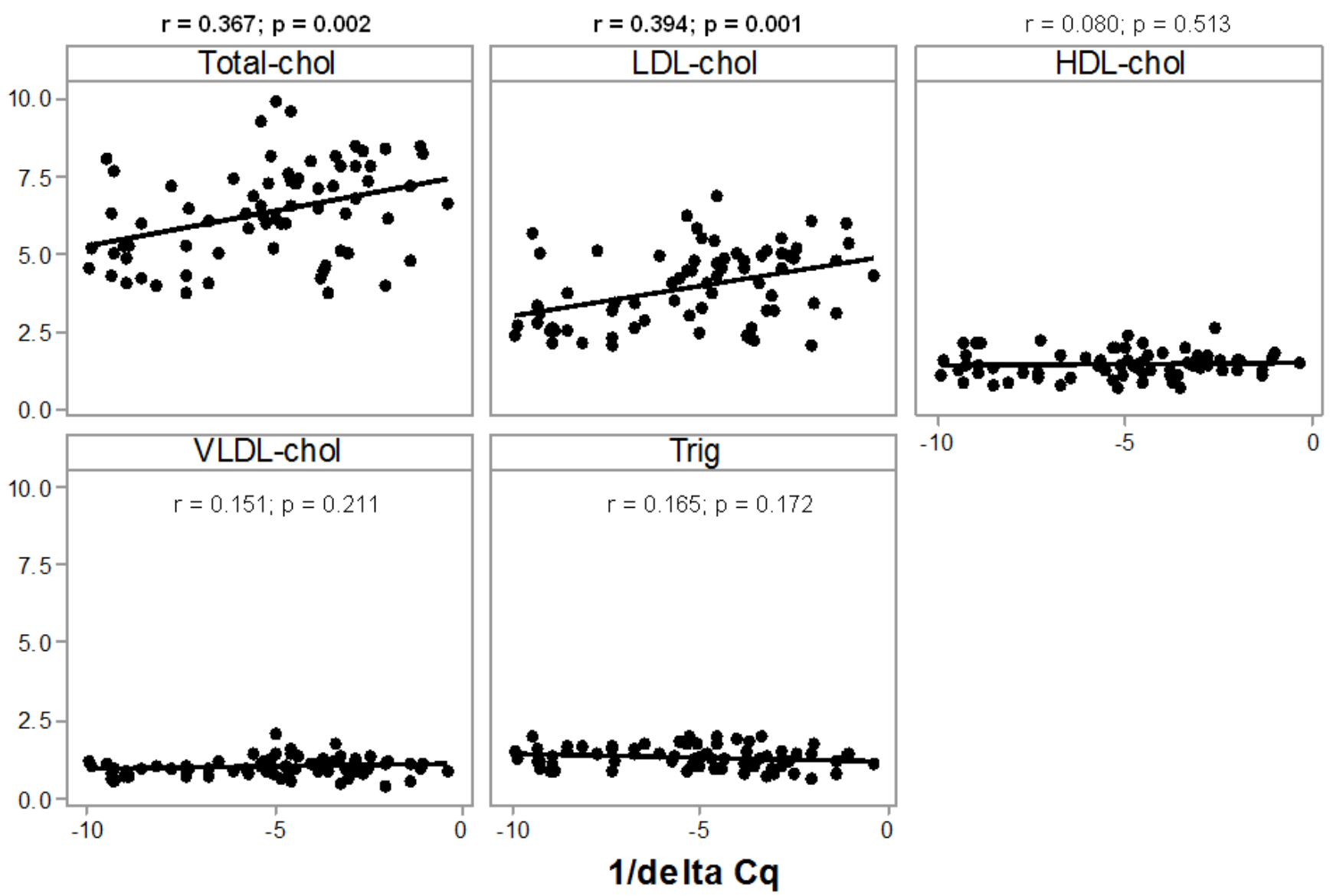

Figure 2 

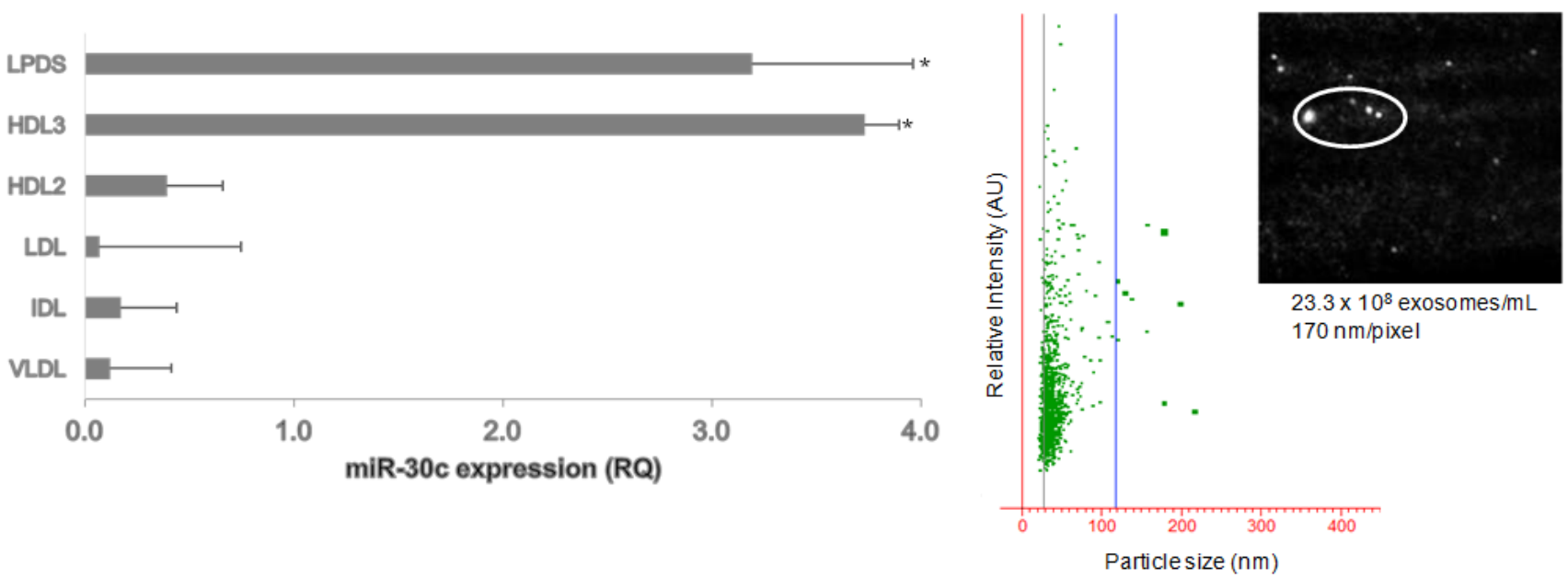

Figure 3 
A

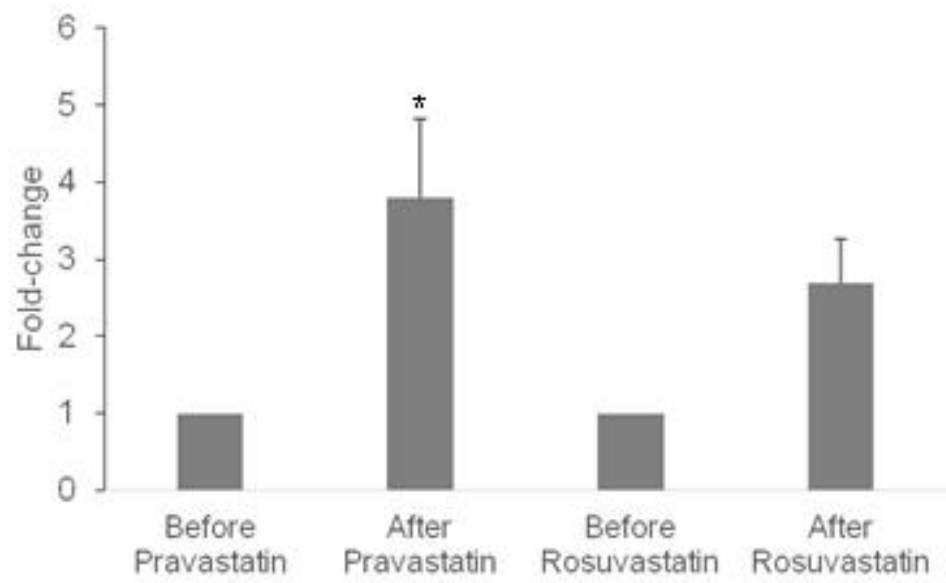

B

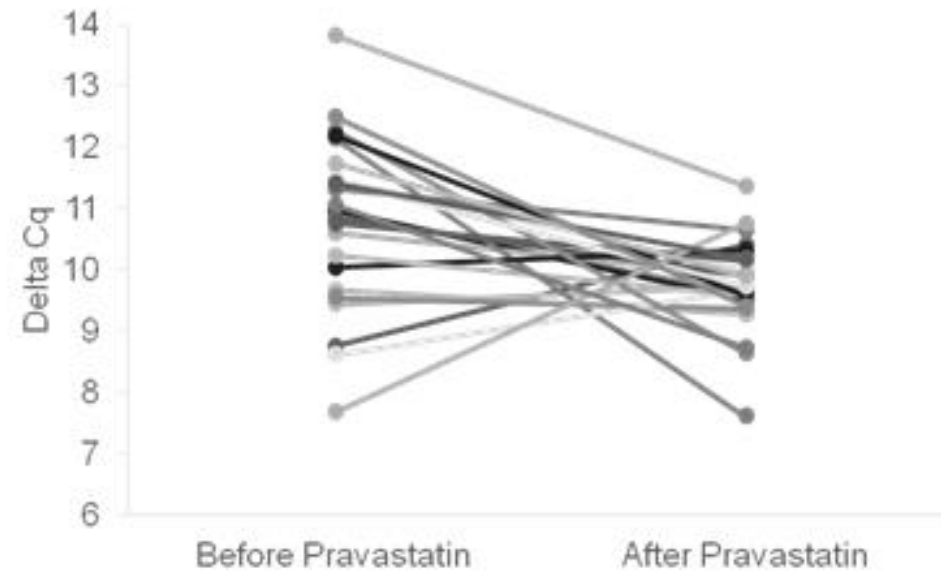

C

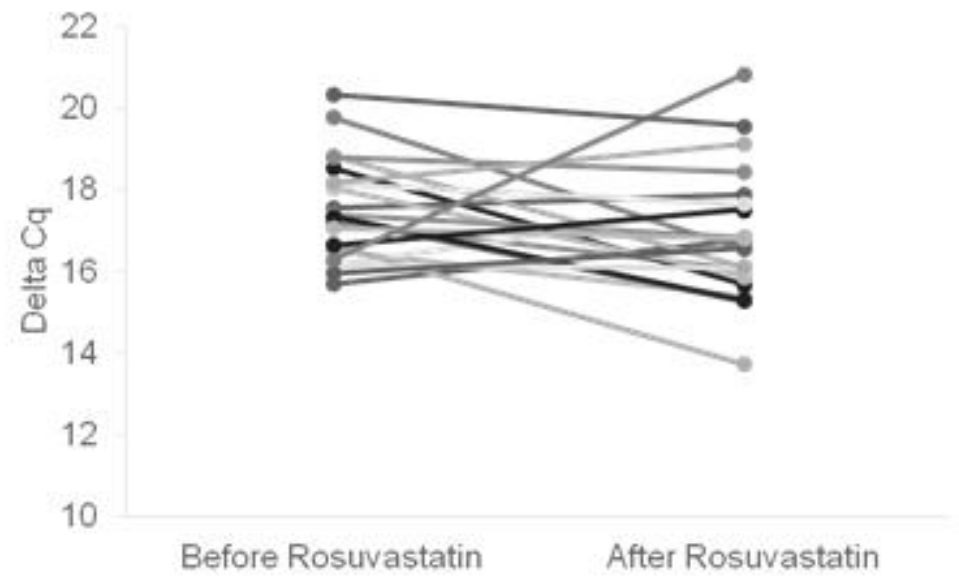

Figure 4 


\section{Highlights}

This study highlights the following for the first time:

- $\quad$ miR-30c is positively correlated with total- and LDL-cholesterol implicating specific regulatory functions in lipid homeostasis.

- We show that specific micro-RNAs are transported differently in the circulation with miR-30c being transported in both exosomes and on HDL3.

- $\quad$ This study shows that pravastatin significantly increased circulating miR-30c expression adding to the pleiotropic dimensions of statins and demonstrating that circulating $\mathrm{miR}$ is amenable to therapeutic manipulation. 